\title{
Anti-hyperalgesic effects of AG490, a Janus kinase inhibitor, in a rat model of inflammatory pain
}

\author{
BOPAIAH P. CHEPPUDIRA, THOMAS H. GARZA, LAWRENCE N. PETZ, \\ JOHN L. CLIFFORD and MARCIE FOWLER \\ U.S. Army Institute of Surgical Research, Battlefield Pain Management Research Task Area, \\ Fort Sam Houston, TX 78234, USA
}

Received May 19, 2015; Accepted July 3, 2015

DOI: $10.3892 /$ br.2015.497

\begin{abstract}
Interleukin 6 (IL-6) has a critical role in pain mechanisms. IL-6 signals through the Janus-activated kinases 2 (JAK2) and signal transducer and activator of transcription 3 (STAT3) pathway. The contribution of JAK2 signaling in inflammation-induced hyperalgesia has not been addressed previously. The role of this pathway was investigated using the JAK2 inhibitor, AG490, in a rat model of inflammatory pain. Unilateral hind paw inflammatory pain was induced in male Sprague-Dawley rats by intraplantar (i.pl.) injection of $3.5 \%$ K-carrageenan. Inflamed rats received an i.pl. injection of either $3.5 \%$ of dimethylsulfoxide or AG490 (1-10 $\mu \mathrm{g})$. The antinociceptive effects of AG490 were assessed by 2 pain behavioral assays $4 \mathrm{~h}$ later: The thermal and mechanical hyperalgesia tests. AG490 (1-10 $\mu \mathrm{g})$ significantly attenuated $\kappa$-carrageenan-induced thermal hyperalgesia in a dose-dependent manner. AG490 also reduced mechanical hyperalgesia. Co-administration of opioid receptor antagonist naloxone $(10 \mu \mathrm{g})$ and AG490 (10 $\mu \mathrm{g})$ did not reverse AG490-produced antinociceptive activity, suggesting that the $\mu$-opioid receptor is not responsible for the anti-hyperalgesic effects of AG490. Therefore, we suggest that AG490 produces these effects by blocking JAK2 signaling. In conclusion, JAK2 inhibitors may represent a novel class of non-narcotic drugs to treat inflammatory pain.
\end{abstract}

\section{Introduction}

Inflammatory disease is associated with immune cell migration, cytokine release, edema, erythema, pain, allodynia and hyperalgesia (1), and controlling inflammatory pain symptoms is a major clinical problem. The interventions for the effective

Correspondence to: Dr Bopaiah P. Cheppudira, U.S. Army Institute of Surgical Research, Battlefield Pain Management Research Task Area, 3698 Chambers Pass, Fort Sam Houston, TX 78234, USA

E-mail: bopaiah.p.cheppudira.vol@mail.mil

Key words: inflammatory pain, Janus kinase 2 inhibitor, AG490, thermal hyperalgesia, mechanical hyperalgesia treatment of inflammatory pain in patients that are refractory to available opioid and non-opioid analgesics, or who develop serious side effects causing drug withdrawal are currently unclear. These concerns have prompted the identification of novel targets and experimental strategies to test potent interventions in well-validated animal models of inflammatory pain.

Interleukin 6 (IL-6) is a pleiotropic cytokine produced by different types of immune cells. Its level is markedly upregulated in various pathological conditions. IL-6 possesses pro-inflammatory and pro-nociceptive activities $(2,3)$. Multiple animal studies have shown that administration of IL- 6 through intraplantar, intrathecal and intracerebroventricular routes induces allodynia or hyperalgesia (4). IL-6 mediates its action by binding to the receptor IL-6R, which in turn associates with the membrane-bound protein gp130. The IL-6/IL-6R complex initiates an intracellular cascade of phosphorylation of several signaling proteins, including those in the Janus-activated kinases 2 (JAK2) and signal transducer and activator of transcription 3 (STAT3) pathway. A number of previous studies have also shown that inhibition of IL-6 signaling is a promising approach to treat pain (5-7).

AG490 is a synthetic derivative of benzylidenemalononitrile. It is a specific and potent inhibitor of JAK2 signaling (8). Although a few studies have shown that AG490 possesses anti-hyperalgesic and anti-allodynic effects, its role in the mediation or inhibition of inflammatory pain, and the mechanism by which it may do so, is not clearly understood.

The $K$-carrageenan-induced inflammatory pain model is widely used to study acute effects of analgesic drugs (9). The antinociceptive activity of AG490 was evaluated using this model. Previous studies have shown that IL-6 reduces hyperalgesia by increasing spinal $\mu$-opioid receptor during chronic inflammation $(10,11)$ and also releases opioid peptides from immune cells $(12,13)$. Therefore, whether the antinociceptive activity of AG490 involves the opioid system at peripheral site was also investigated.

\section{Materials and methods}

Animals. A total of 28 Male Sprague-Dawley rats (250-300 g were used) were housed (2/cage) under standard conditions (12:12-h light:dark cycle with ad libitum access to food and 
water). The study was conducted in compliance with the Animal Welfare Act, the implementing Animal Welfare Regulations and the principles of the Guide for the Care and Use of Laboratory Animals.

Drugs. On the day before administering $\Lambda$-carrageenan (Sigma-Aldrich, St. Louis, MO, USA), it was dissolved in saline to create a $3.5 \%$ solution and stored at $4^{\circ} \mathrm{C}$. AG490 (Sigma-Aldrich) was dissolved in $3.5 \%$ dimethylsulfoxide (DMSO) prior to the start of an experiment on each study. Naloxone methiodide (Sigma-Aldrich) was dissolved in water, and DMSO was diluted with water.

Induction of inflammatory pain. Unilateral hind paw inflammation in the rat was induced by intraplantar (i.pl.) injection of $100 \mu 13.5 \% \mathrm{~K}$-carrageenan in the left hind paws (9). Inflammation was evident at $48 \mathrm{~h}$ post-injection, as indicated by redness and swelling of the affected paw.

Experimental groups, design and treatment. The experiments were performed in rats $48 \mathrm{~h}$ after $\Lambda$-carrageenan injection. A total of 4 groups $(n=6)$ of rats were randomly included in the dose-response study. Group 1 was the vehicle control, which received $100 \mu \mathrm{l}$ i.pl. injection of $3.5 \%$ DMSO in saline. Groups 2-4 were injected with 3 different doses of AG490 (1, 5 or $10 \mu \mathrm{g})$. To study the effects of naloxone on AG490-induced antinociception, an additional group of rats (group 5; n=4) was observed. Group 5 was co-administered with AG490 $(10 \mu \mathrm{g})$ and naloxone $(10 \mu \mathrm{g})$. The drugs were administered i.pl. in a volume of $100 \mu \mathrm{l}$. As reported earlier, the in vivo pharmacological effects of AG490 were observed $4 \mathrm{~h}$ after treatment $(5,6)$. Thus, the behavioral tests were performed before (baseline assessment) and $4 \mathrm{~h}$ after treatment. First, the rats were subjected to the thermal hyperalgesia test; $10 \mathrm{~min}$ later, the paw pressure test was performed on the same set of rats. All the experiments were performed between 8:00 a.m. and 2:00 p.m. to reduce the confounding influence of diurnal variations, and all the procedures were performed in a blinded fashion.

Thermal hyperalgesia test. The thermal hyperalgesia test was performed using a plantar analgesia instrument (IITC Life Science, Woodland Hills, CA, USA), as described previously (6). Briefly, rats were acclimated to the Plexiglas chambers for $30 \mathrm{~min}$ prior to testing. A radiant heat source was focused at the mid-plantar surface of the hind paw, and the paw withdrawal latency (PWL) was recorded. Both paws were tested alternatively at 2-3 min intervals for a total of 3 trials. A mean count and latency were used for analysis. The percentage of maximal possible effect (MPE) (\%) was calculated according to the following formula: $\% \mathrm{MPE}=$ (post-drug latency - pre-drug latency)/[cut-off (20 sec) - pre-drug latency] x 100 .

Paw pressure test. The nociceptive thresholds were assessed using a modified digital Randall-Selitto device (IITC Life Science). Briefly, each rat was gently restrained and an increasing mechanical pressure was exerted on the mid-plantar surface of the hind paw until a paw withdrawal response was observed. A total of 3 trials were performed on the inflamed and contralateral paws, and the average was taken for analysis. The paw withdrawal threshold (PWT) was expressed in grams, and a cut-off value of $300 \mathrm{~g}$ was used to prevent tissue injury (14). PWT was calculated for each rat using $\triangle \mathrm{PWT}=$ tested $\mathrm{PWT}$ - baseline PWT (5).

Data Analysis. The data are expressed as mean \pm standard error of the mean. The difference in PWL and PWT between ipsilateral (inflamed) and contralateral (non-inflamed) paws were compared using a paired t-test. The results of dose-response studies and naloxone effects were evaluated by analysis of variance followed by Bonferroni post hoc test $(\mathrm{P}<0.05$ was considered to indicate a statistically significant difference).

\section{Results}

$\kappa$-carrageenan treatment induces thermal and mechanical hyperalgesia. As reported previously (9), $K$-carrageenan treatment to the left hind paw produced localized inflammation, as evidenced by redness and swelling. In comparison to the contralateral paws, the inflamed paws showed a significant decrease in PWL and PWT to the application of thermal and mechanical stimuli ( $\mathrm{P}<0.05$; data not shown).

AG490 inhibits thermal hyperalgesia and reduces mechanical hyperalgesia. An i.pl. injection of AG490 (1,5 and $10 \mu \mathrm{g})$ to the inflamed paw produced a dose-dependent, statistically significant anti-hyperalgesic effect in the thermal test (Fig. 1A). Bonferroni post-hoc analysis showed a significant difference between control and $5 \mu \mathrm{g}(\mathrm{P}<0.05)$, and control and $10 \mu \mathrm{g}$ of the AG490-treated groups $(\mathrm{P}<0.01)$. Although the $1 \mu \mathrm{g}$ AG490-treated group showed a trend of increased PWL compared to the control group, it did not reach significance $(\mathrm{P}>0.05)$. The most potent effect was observed for $10 \mu \mathrm{g}$ of AG490 in comparison to the control group. AG490 treatment did not alter the PWL of the contralateral paws ( $P>0.05$, data not shown). These results clearly indicate that blocking JAK2 signaling attenuates thermal hypersensitivity in an inflamed condition. In the mechanical hyperalgesia test, AG490 treatment resulted in a trend towards increased PWT when compared to the control group (Fig. 1B), but it was not of statistical significance $(\mathrm{P}=0.0504)$. No difference in PWT was observed on contralateral paws $(\mathrm{P}>0.05$, data not shown).

Naloxone treatment did not alter AG490-induced thermal and mechanical hyperalgesia. Co-administration of naloxone and AG490 to the inflamed paw increased the PWL in comparison to AG490 and control groups $(\mathrm{P}<0.01)$ (Fig. 2A). This suggests that naloxone had no effect on the antinociceptive activity of AG490. Notably, the animals that received AG490 and naloxone exhibited greater nociceptive effects compared to the animals treated with AG490 alone; however, the difference between the groups was not significant $(\mathrm{P}>0.05)$. A similar trend was observed in the mechanical hyperalgesia test (Fig. 2B) without a significant difference between groups $(\mathrm{P}>0.05)$. From these data, the opioid signaling system appears to not be involved in AG490-mediated antinociception. 
A

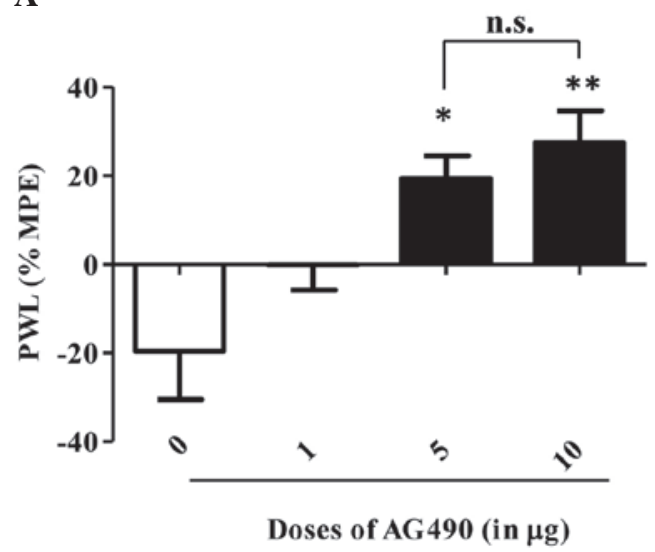

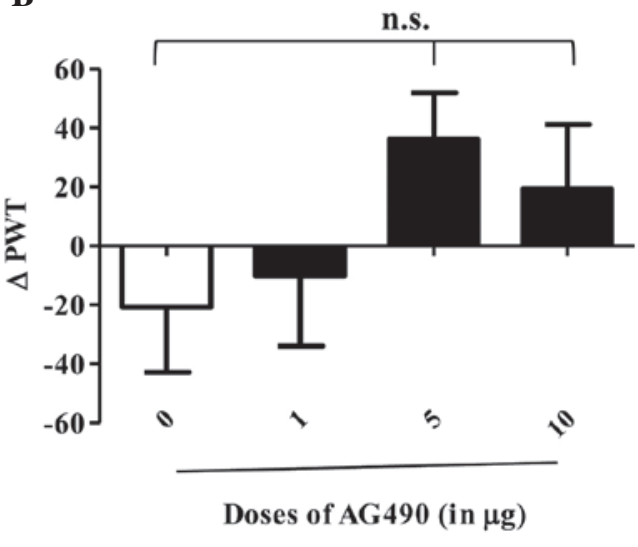

Figure 1. (A) Intraplantar injection of AG490 dose-dependently increases the paw withdrawal response (PWL) to a thermal stimulus and (B) paw withdrawal threshold (PWT) to a mechanical stimulus. The thermal hyperalgesia data are presented as a mean percentage of maximal possible effect (\% MPE) \pm standard error of the mean (SEM). Mechanical hyperalgesia data are expressed by $\triangle \mathrm{PWT} \pm \mathrm{SEM}$. "P<0.05 vs. control; ${ }^{* *} \mathrm{P}<0.001$ vs. control. "Indicates significant difference and n.s. represents not significant.

A

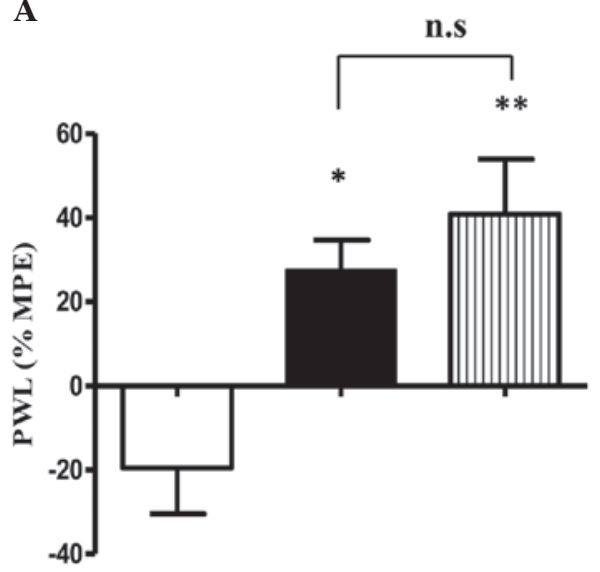

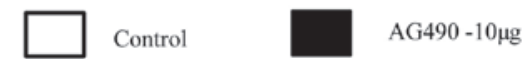

B

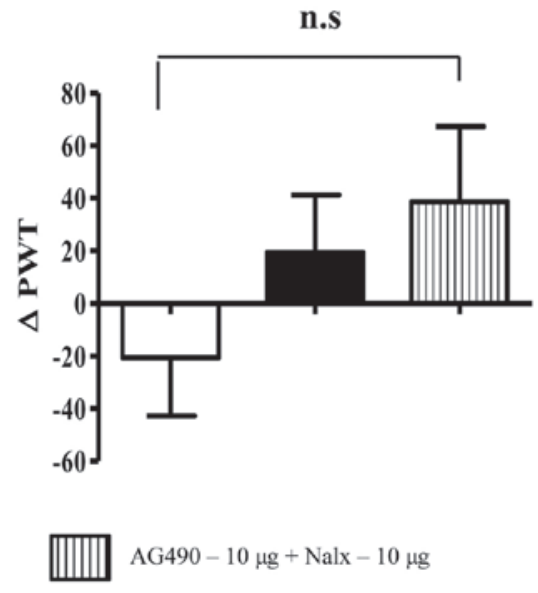

Figure 2. (A) Intraplantar co-administration of AG490 and naloxone increases the paw withdrawal response (PWL) to a thermal stimulus and (B) paw withdrawal threshold (PWT) to a mechanical stimulus. The thermal hyperalgesia data are presented as a mean percentage of maximal possible effect $(\% \mathrm{MPE}) \pm$ standard error of the mean $(\mathrm{SEM})$. Mechanical hyperalgesia data are expressed by $\Delta \mathrm{PWT} \pm \mathrm{SEM}$. ${ }^{*} \mathrm{P}<0.05$ vs. control; ${ }^{* *} \mathrm{P}<0.001 \mathrm{vs}$. control. *Indicates significant difference and n.s. represents not significant.

\section{Discussion}

In the present study, the behavioral assays showed that the JAK2 signaling inhibitor AG490, was effective in attenuating thermal hyperalgesia and reducing mechanical hyperalgesia in the $K$-carrageenan-induced inflammatory pain model. Furthermore, the data indicates that AG490-mediated antinociceptive activity is independent of the opioid system.

Tyrosine kinase inhibitors, including AG490, are effective for the treatment of various malignancies. Previous studies have shown that blocking JAK activity with AG490 inhibits STAT3. Coinciding with this effect was the observation of reduced levels of cytokine production, inflammatory cell infiltration and nitric acid production $(8,15)$. In addition, previous studies have demonstrated that administration of AG490 attenuates mechanical allodynia and/or thermal hyperalgesia in painful diabetic neuropathy, peripheral nerve injury and in the cyclophosphamide-induced bladder pain model $(5,6,16)$.
The present findings are in agreement with these studies. Acute administration of AG490 dose-dependently attenuates thermal hyperalgesia. Additionally, in the mechanical hyperalgesia test, AG490 treatment increased the PWT at all doses tested; however, this effect was not statistically significant. Therefore, we speculate that AG490 is more effective at reducing mechanical allodynia as measured in previous studies $(5,6)$, than for reducing mechanical hyperalgesia, as shown in the present study. It is also possible that differences in animal model, and route and dose of AG490 administration influence the effects of AG490 on the response to mechanical stimuli.

There are reports demonstrating that the cytokine IL-6 acts at the site of inflammation on resident and circulating immune cells to cause the release of endogenous opioids, which subsequently activate peripheral opioid receptors to produce analgesia $(12,13)$. Whether the antinociceptive activity of AG490 is mediated by release of endogenous 
opioid peptides was indirectly investigated through the use of the opioid receptor antagonist, naloxone. To the best of our knowledge, the data, for the first time, showed that naloxone did not reverse the AG490-mediated increase in PWL and PWT. This indicates that AG490 produced antinociceptive activity through a non-opioid mechanism. Of note, in the thermal and mechanical sensory sensitivity tests, AG490 and naloxone-treated animals showed increased antinociceptive activity compared to animals receiving AG490 alone. Further studies are required to investigate the mechanisms of this effect.

In conclusion, acute administration of AG490 is effective in reducing $\Lambda$-carrageenan-induced thermal hyperalgeisa, and the effect is likely to be specifically mediated through the JAK2 signaling pathway without involvement of the opioid system. Further investigation is warranted to determine whether AG490 could be a novel non-narcotic drug for the management of chronic pain associated with inflammatory disease.

\section{Acknowledgements}

The present study was supported by the United States Army Medical Research and Material Command Combat Causality Care Research and the Clinical and Rehabilitative Medicine Research programs. Dr Cheppudira was supported by the National Research Council Senior Research Associate Fellowship.

\section{References}

1. Ji RR, Xu ZZ and Gao YJ: Emerging targets in neuroinflammation-driven chronic pain. Nat Rev Drug Discov 13: 533-548, 2014.

2. Wojdasiewicz P, Poniatowski LA and Szukiewicz D: The role of inflammatory and anti-inflammatory cytokines in the pathogenesis of osteoarthritis. Mediators Inflamm 2014: 561459, 2014

3. Oka T, Oka K, Hosoi M and Hori T: Intracerebroventricular injection of interleukin- 6 induces thermal hyperalgesia in rats. Brain Res 692: 123-128, 1995.
4. De Jongh RF, Vissers KC, Meert TF, Booij LH, De Deyne CS and Heylen RJ: The role of interleukin-6 in nociception and pain. Anesth Analg 96: 1096-1103, 2003.

5. Dominguez E, Rivat C, Pommier B, Mauborgne A and Pohl M: JAK/STAT3 pathway is activated in spinal cord microglia after peripheral nerve injury and contributes to neuropathic pain development in rat. J Neurochem 107: 50-60, 2008.

6. Cheppudira BP, Girard BM, Malley SE, Dattilio A, Schutz KC May V and Vizzard MA: Involvement of JAK-STAT signaling/function after cyclophosphamide-induced bladder inflammation in female rats. Am J Physiol Renal Physiol 297: F1038-F1044, 2009.

7. Guptarak J, Wanchoo S, Durham-Lee J, Wu Y, Zivadinovic D, Paulucci-Holthauzen A and Nesic O: Inhibition of IL-6 signaling: A novel therapeutic approach to treating spinal cord injury pain. Pain 154: 1115-1128, 2013.

8. Levitzki A: Tyrphostins - potential antiproliferative agents and novel molecular tools. Biochem Pharmacol 40: 913-918, 1990.

9. Radhakrishnan R and Sluka KA: Spinal muscarinic receptors are activated during low or high frequency TENS-induced antihyperalgesia in rats. Neuropharmacology 45: 1111-1119, 2003.

10. Tekieh E, Zaringhalam J, Manaheji H, Maghsoudi N, Alani B and Zardooz H: Increased serum IL-6 level time-dependently regulates hyperalgesia and spinal mu opioid receptor expression during CFA-induced arthritis. EXCLI J 10: 23-33, 2011.

11. Zaringhalam J, Tekieh E, Manaheji $\mathrm{H}$ and Akhtari Z: Cellular events during arthritis-induced hyperalgesia are mediated by interleukin-6 and p38 MAPK and their effects on the expression of spinal mu-opioid receptors. Rheumatol Int 33: 2291-2299, 2013.

12. Członkowski A, Stein C and Herz A: Peripheral mechanisms of opioid antinociception in inflammation: Involvement of cytokines. Eur J Pharmacol 242: 229-235, 1993.

13. Bianchi M, Maggi R, Pimpinelli F, Rubino T, Parolaro D, Poli V, Ciliberto G, Panerai AE and Sacerdote P: Presence of a reduced opioid response in interleukin-6 knock out mice. Eur J Neurosci 11: 1501-1507, 1999.

14. Gainok J, Daniels R, Golembiowski D, Kindred P, Post L, Strickland R and Garrett N: Investigation of the anti-inflammatory, antinociceptive effect of ellagic acid as measured by digital paw pressure via the Randall-Selitto meter in male Sprague-Dawley rats. AANA J 79 (Suppl 4): S28-S34, 2011.

15. Dimitrova P and Ivanovska N: Tyrphostin AG-490 inhibited the acute phase of zymosan-induced inflammation. Int Immunopharmacol 8: 1567-1577, 2008.

16. Kou ZZ, Li CY, Tang J, Hu JC, Qu J, Liao YH, Wu SX, Li H and Li YQ: Down-regulation of insulin signaling is involved in painful diabetic neuropathy in type 2 diabetes. Pain Physician 16: E71-E83, 2013 